\title{
Z-ISO: a catching up step towards formation of colored carotenoids in tomatoes
}

\author{
Efremov G.I.*, Shchennikova A.V., Kochieva E.Z. \\ Federal Research Centre "Fundamentals of Biotechnology" RAS, Moscow, Russia \\ *email: gleb_efremov@mail.ru
}

Carotenoid biosynthesis in fleshy fruits has several critical steps, one of which is the isomerization of 9,15,9'-tri-cis- $\zeta$-carotene, mediated by Z-ISO (15-cis- $\zeta$-carotene isomerase). This enzyme is considered a gatekeeper of the carotenoid pathway, especially in tissues without light exposure. In total, 20 complete Z-ISO homologs, including the 5'-UTR and the promotor, were identified in 12 Solanum lycopersicum cultivars and eight accessions of wild tomato species $S$. pimpinellifolium, S. cheesmaniae, $S$. chilense, $S$. habrochaites, and $S$. pennellii, differing in fruit color and carotenoid content. Compared to the Z-ISO of cv. Heinz 1706, the variability of the identified genes was $14.98 \%$ (complete sequences), $10.36 \%$ (exons), $21.50 \%$ (5'-UTR), and $17.89 \%$ (promoter). All putative Z-ISO proteins were 369 aa and contained a 58-aa transit peptide and a 15-cis-zeta-carotene isomerase domain. Comparative structural analysis of Z-ISO homologs identified 20 conserved motifs, 10 of which were common, and the other 10 were species-specific. Z-ISO enzymes of analyzed accessions contained 24 radical aa substitutions compared to cv. Heinz Z-ISO. Z-ISO expression was analyzed in leaves and fruits (immature green, mature green, breaker, and ripe) of S. lycopersicum cv. Heinz, Korneevskii, and Kopilka zheltaya, as well as $S$. cheesmaniae, S. habrochaites and $S$. pennellii accessions. The expression level of Z-ISO was found to correlate with the color and carotenoid content of the fruit. In fruits of red-fruited cv. Heinz and Korneevskii with abundant lycopene and yellow-fruited cv. Kopilka zheltaya with high $\beta$-carotene content, Z-ISO transcription was 10-20 times higher than in S. cheesmaniae pale yellow fruits with $\beta$-carotene traces and a lack of lycopene at the breaker and ripe stages respectively. The lowest expression was in fruits of green-fruited $S$. habrochaites and $S$. pennellii. In leaves, Z-ISO expression was significantly higher in cv. Heinz than in other cultivars and species, where it remained low or absent.

Acknowledgments: This study was supported by the Russian Science Foundation (grant No. 19-16-00016), and, partially, by the Ministry of Science and Higher Education of the Russian Federation. 